\title{
Applicability of the Adjoint Sensitivity-Based Data Assimilation Method: Radar Data Assimilation for Heavy Rainfall Cases over the Korean Peninsula
}

\author{
Yonghan Choi, Gyu-Ho Lim, and Dong-Kyou Lee \\ School of Earth and Environmental Sciences, Seoul National University, Seoul, Korea
}

\begin{abstract}
In this study, the usefulness of the Adjoint Sensitivity-based Data Assimilation (ASDA) method in assimilating radar data was investigated by comparing it with the Three/Four Dimensional Variational (3/4D-Var) method. A total of 10 heavy rainfall cases over the Korean Peninsula were selected and classified as one of four Heavy Precipitation Systems (HPSs) according to their phenomenological properties.

The Quantitative Precipitation Forecasting (QPF) skill is evaluated by computing the threat and bias scores, and the Root Mean Square Errors (RMSEs) of the simulated radial velocity are also calculated. The forecast skill of the ASDA method is comparable to that of the 4D-Var method in most of cases. This is because the ASDA method has some of the advantages of the 4D-Var method such as flow-dependent background error covariance and well-balanced analysis. In addition, the dependence of the QPF skill of the ASDA method on the characteristics of heavy rainfall cases is analyzed by calculating time-lagged autocorrelations of the observed radar data.

(Citation: Choi, Y., G.-H. Lim, and D.-K. Lee, 2015: Applicability of the adjoint sensitivity-based data assimilation method: Radar data assimilation for heavy rainfall cases over the Korean Peninsula. SOLA, 10, 53-58, doi:10.2151/sola.2015-012.)
\end{abstract}

\section{Introduction}

Data assimilation in meteorology is the process through which all the available information is used to estimate the state of the atmospheric flow as accurately as possible (Talagrand 1997). The Four Dimensional Variational (4D-Var) data assimilation method is the one of the most advanced data assimilation methods in meteorology. In the 4D-Var method, observations can be assimilated at the time of their measurement; flow-dependent background error covariance is implicitly used; a forecast model is used as a constraint. Despite the merits of the 4D-Var method, it requires a large amount of computing resources due to its iterative minimization of the cost function.

Huang et al. (1997) proposed the Poorman's variational assimilation (PMV) method, where the main idea is to use the adjoint model to produce an improved first guess, which leads to an improved Optimal Interpolation (OI) analysis. However, the PMV violates the basic assumption of data assimilation, that the first guess and observation errors should be uncorrelated, although the orthogonality between the barotropic OI analysis and the baroclinic PMV analysis alleviates the violation.

Thépaut et al. (1996) investigated dynamical structure functions of the 4D-Var method by analyzing the analysis increments produced by one single observation. They found a strong link between the analysis increments of the 4D-Var method and the singular vectors of the tangent linear dynamics computed over the same time interval. Several studies noticed that the adjoint sensitivity can be expressed as the linear combination of the singular vectors (Gelaro et al. 1998; Kim et al. 2004; Jung and Kim 2009). Therefore, the adjoint sensitivity can be used as a perturbation to

Corresponding author: Yonghan Choi, School of Earth and Environmental Sciences, Seoul National University, 1 Gwanak-ro, Gwanak-gu, Seoul 151-742, Korea. E-mail: cyh082@snu.ac.kr. (C2015, the Meteorological Society of Japan. improve initial conditions.

Choi et al. (2014) proposed the Adjoint Sensitivity-based Data Assimilation (ASDA) method using the adjoint sensitivity of forecast error and the objectively-defined scaling factor, and they compared the ASDA method with existing variational data assimilation methods for a heavy rainfall case over the Korean Peninsula. In the ASDA method, the first guess and observation errors are not correlated, and the computational cost is significantly reduced compared to the 4D-Var method. In this study, the ASDA method is applied to various types of heavy rainfall over the Korean Peninsula to obtain statistical robustness of the conclusions of Choi et al. (2014). Mathematical equations related to the ASDA method are summarized in Section 2, and experimental design and heavy rainfall cases are described in Section 3. Section 4 includes results and discussions, and finally, Section 5 contains summary and conclusions.

\section{Adjoint Sensitivity-based Data Assimilation (ASDA) method}

In this section, we briefly introduce the mathematical formulation of the ASDA method, and a schematic diagram for the ASDA procedure is shown in Fig. $1 \mathrm{~b}$. A full description can be found in Choi et al. (2014).

In the ASDA method, the adjoint sensitivity of forecast error is used as a perturbation to improve the original first guess.

$$
\boldsymbol{\delta} \mathbf{x}_{\mathbf{0}}^{\mathrm{fg}}=\alpha_{o p t} \mathbf{A}^{-1} \frac{\partial R}{\partial \mathbf{x}_{\mathbf{0}}}
$$

where aopt is an optimal scaling factor $\mathbf{x}$ is an initial condition, $\mathbf{A}^{-1}$ is for a unit conversion from the adjoint sensitivity to the state vector, and $R$ is a response function of forecast error. Therefore, the right-hand side of the above equation represents the scaled adjoint sensitivity of forecast error, and this is used as the perturbation denoted by $\delta \mathbf{x}^{\mathrm{fg}}$.

The adjoint sensitivity of forecast error scaled by the optimal scaling factor is added to the original first guess to make an improved first guess, and 3D-Var analysis is conducted using the improved first guess to make the final analysis of the ASDA method.

$$
\begin{aligned}
& \mathbf{x}_{\mathbf{0}}^{\text {new }}=\mathbf{x}_{\mathbf{0}}+\boldsymbol{\delta} \mathbf{x}_{\mathbf{0}}^{\mathrm{fg}}=\mathbf{x}_{\mathbf{0}}+\alpha_{o p t} \mathbf{A}^{-1} \frac{\partial R}{\partial \mathbf{x}_{\mathbf{0}}} \\
& \mathbf{x}_{\mathbf{0}}^{\text {ASDA }}=\mathbf{x}_{\mathbf{0}}^{\text {new }}+\boldsymbol{\delta} \mathbf{x}_{\mathbf{0}}^{\text {ASDA }}
\end{aligned}
$$

where, $\mathbf{x}$ is the original first guess, $\mathbf{x}^{\text {new }}$ is the improved first guess, $\delta \mathbf{x}^{\text {ASDA }}$ is the 3D-Var analysis increment based on the improved first guess, and $\mathbf{x}^{\text {ASDA }}$ is the final analysis of the ASDA method. It is noted that the final 3D-Var analysis is conducted at $t=0 \mathrm{~min}$ to avoid using the observation at some forecast time (here, at $t=$ $30 \mathrm{~min}$ ) twice.

\section{Experimental design}

In this study, we used the Advanced Research Weather Research and Forecasting (ARW-WRF) model version 3.5.1 (Skamarock et al. 2008), with the same domain configuration and physical parameterizations as in Choi et al. (2014). Detailed 


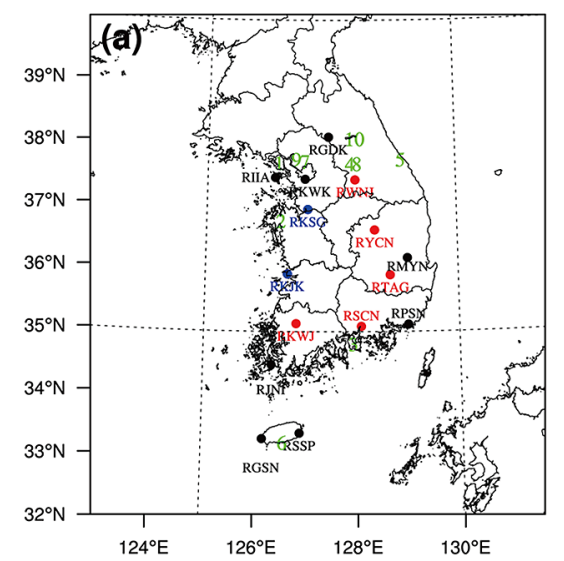

(b)

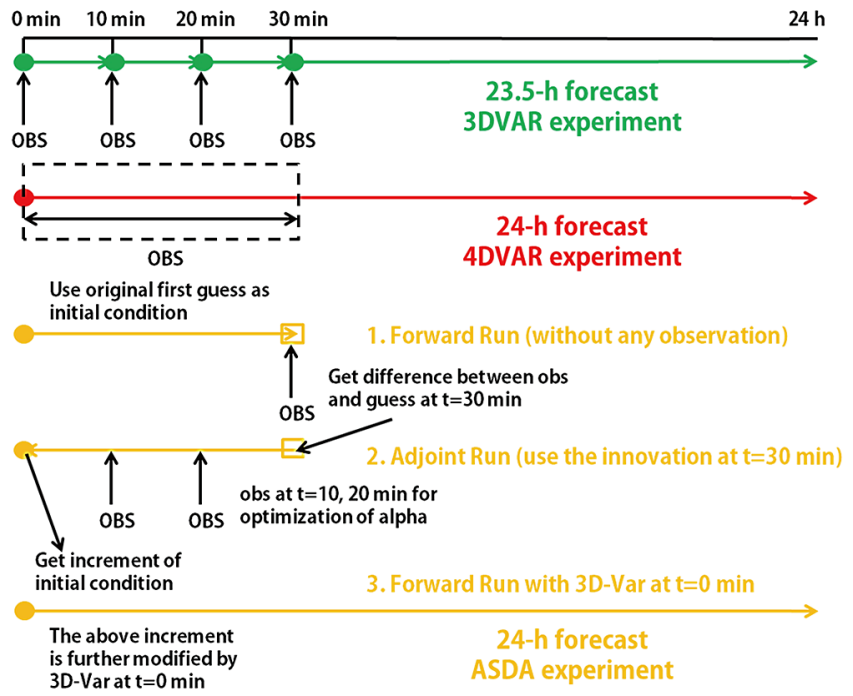

Fig. 1. (a) Locations of radar observation sites operated by the Korea Meteorological Administration (black), the Korea Air Force (red), and the United States Air Force (blue). Locations of maximum rainfall points for 10 heavy rainfall cases are also shown with the corresponding case numbers. (b) A schematic diagram for design of data assimilation experiments. information on the domain configuration and physical parameterizations is given in Table 1. We also utilized version 3.5.1 of the WRF Data Assimilation (WRFDA; Barker et al. 2012; Huang et al. 2009) system, and data assimilation experiments were conducted only on the 6-km domain. Radar radial velocity data from 15 radar observation sites over the Korean Peninsula (Fig. 1a) were assimilated using the 3D-Var, 4D-Var, and ASDA methods. Information on the background, background error covariance, observation, and observation error covariance is summarized in Table 1, and details can be found in Choi et al. (2014).

A total of 10 heavy rainfall cases over the Korean Peninsula were selected, and each heavy rainfall case was classified as Isolated Thunderstorm (IS), Convection Band (CB), Cloud Cluster (CC), or Squall Line (SL) according to Lee and Kim (2007). A summary of the heavy rainfall cases is given in Table 2, and the geographical locations of the maximum rainfall points are shown in Fig. 1a. For each case, four experiments were conducted: CONTROL, 3DVAR, 4DVAR, and ASDA experiments (Fig. 1b). In the CONTROL experiment, no radar data were assimilated, and 24-h forecast ${ }^{1}$ from the analysis time was made. In the 3DVAR experiment, radar data were assimilated using the 3D-Var method in cycling mode for a fair comparison with the 4D-Var and ASDA methods. Radar data were combined with the background every 10 minutes and this led to the 3D-Var analysis. Following the analysis, 10-min forecast was made and this was used as the background for the next cycle. This analysis-forecast cycle repeated during a 30 -minute period, and finally 23.5 -h forecast was made. Radar data were assimilated using the 4D-Var (ASDA) method in the 4DVAR (ASDA) experiment. In the 4DVAR and ASDA experiments, assimilation of radar data, which were available every 10 minutes within the 30-minute assimilation window, resulted in the analysis, and 24-h forecast was made.

\section{Results and discussion}

Figure 2 shows the threat and bias scores of 12-h accumulated rainfall for a $10-\mathrm{mm}$ threshold value, and it also includes the skill scores of 24-h accumulated rainfall for a $25-\mathrm{mm}$ threshold value. The skill scores were computed using Automatic Weather Station

\footnotetext{
${ }^{1}$ Throughout the paper, the word "simulation" would be more appropriate than the word "forecast" because the NCEP FNL data were used as boundary conditions.
}

Table 1. The WRF model configurations and data assimilation settings.

\begin{tabular}{|c|c|c|c|}
\hline & Domain 1 & Domain 2 & Domain 3 \\
\hline Horizontal resolution & $54 \mathrm{~km}$ & $18 \mathrm{~km}$ & $6 \mathrm{~km}$ \\
\hline Number of horizontal grids & $120 \times 102$ & $121 \times 103$ & $121 \times 127$ \\
\hline Number of vertical levels (Model-top pressure) & \multicolumn{3}{|c|}{$35(50 \mathrm{hPa})$} \\
\hline Cumulus parameterization scheme & Kain- $\mathrm{Fr}_{1}$ & in 2004) & None \\
\hline Microphysics scheme & \multicolumn{3}{|c|}{ WRF Single-Moment 6-class (WSM6) scheme (Hong and Lim 2006) } \\
\hline Planetary boundary layer scheme & \multicolumn{3}{|c|}{ Yonsei University (YSU) scheme (Hong et al. 2006) } \\
\hline Longwave radiation scheme & \multicolumn{3}{|c|}{ Rapid Radiative Transfer Model (RRTM) scheme (Mlawer et al. 1997) } \\
\hline Shortwave radiation scheme & \multicolumn{3}{|c|}{ Dudhia scheme (Dudhia 1989) } \\
\hline Land surface model & \multicolumn{3}{|c|}{ Noah Land Surface Model (Tewari et al. 2004) } \\
\hline Initial/boundary conditions & \multicolumn{3}{|c|}{ NCEP FNL data } \\
\hline Background & \multicolumn{3}{|c|}{ Cold start } \\
\hline Background error covariance & \multicolumn{3}{|c|}{ National Meteorological Center (NMC) method (Parrish and Derber 1992) } \\
\hline Observation preprocessing & \multicolumn{3}{|c|}{ Park and Lee (2009) } \\
\hline Observation resolution & \multicolumn{3}{|c|}{ Horizontal resolution: $6 \mathrm{~km}$, vertical resolution: $0.5 \mathrm{~km}$, temporal resolution: 10 minutes } \\
\hline Observation error & \multicolumn{3}{|c|}{$2 \mathrm{~m} \mathrm{~s}^{-1}$} \\
\hline Observation operator & \multicolumn{3}{|c|}{ Xiao et al. (2005) } \\
\hline
\end{tabular}



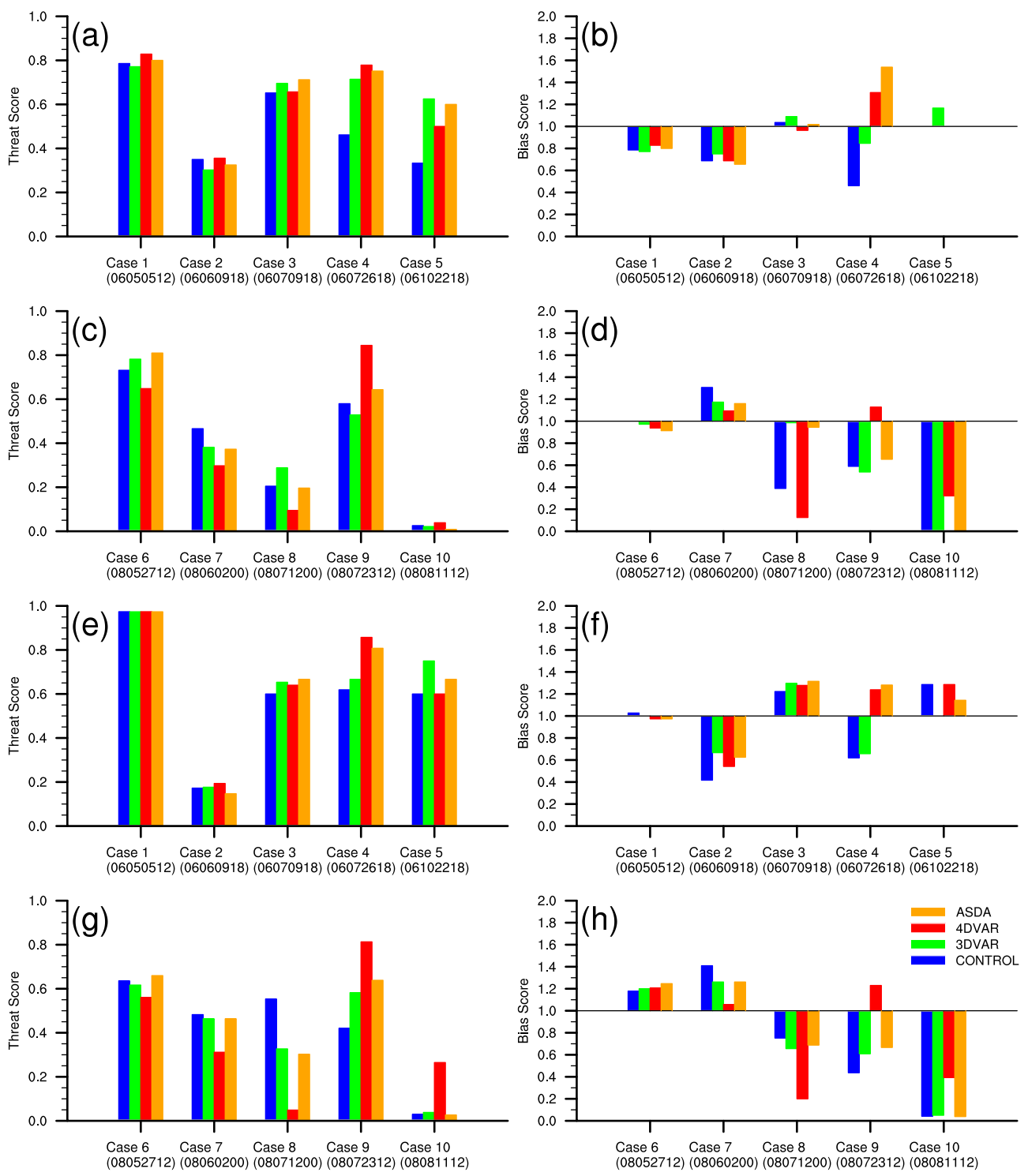

Fig. 2. Skill scores of 12-h (24-h) accumulated rainfall for a threshold value of 10 (25) $\mathrm{mm}$ in the CONTROL (blue), 3DVAR (green), 4DVAR (red), and ASDA (orange) experiments. (a) Threat score and (b) bias score of 12-h accumulated rainfall for Cases 1-5, and (c) threat score and (d) bias score of 12-h accumulated rainfall for Cases 6-10. (e)-(h) Same as (a)-(d) except for 24-h accumulated rainfall.

(AWS) observations over the Korean Peninsula. Although details of the skill scores for the 12-h and 24-h forecasts are different, the overall features for the two forecast ranges are similar. Therefore, for simplicity, the threat and bias scores only for the 24-h forecasts are discussed. The forecast of the ASDA experiment is the most skillful among the experiments in Cases 3 and 6 . The threat and bias scores of the ASDA experiment are comparable to those of the 4DVAR experiment in Cases 4 and 9. In Case 5, the threat and bias scores of the ASDA experiment are better than those of the 4DVAR experiment, but they are worse than those of the 3DVAR experiment. The threat score of the ASDA experiment is lower than that of the CONTROL experiment in Cases 2, 7, 8, and 10. However, it should be noted that the threat score of the ASDA experiment is higher than that of the 4DVAR experiment in Cases 7 and 8. Finally, the threat and bias scores of all experiments are close to one in Case 1. In summary, the Quantitative Precipitation Forecasting (QPF) skill of the ASDA experiment is largely comparable to that of the 4DVAR experiment, and it varies depending on the case.

The Root Mean Square Errors (RMSEs) of radial velocity were calculated using radar radial velocity observations at heights between $3 \mathrm{~km}$ and $7 \mathrm{~km}$ at an interval of one hour, and they were averaged. The averages over the first 12 -h forecasts and the whole 24-h forecasts are shown in Fig. 3. The RMSEs of radial velocity are reduced compared to the CONTROL experiment through the assimilation of radar radial velocity data in all data assimilation experiments regardless of the forecast range (except for Case 1 where the RMSE of the 3DVAR experiment is slightly larger than that of the CONTROL experiment). In all cases except for Case 8, the RMSE of radial velocity of the ASDA experiment is less than that of the 3DVAR experiment. For the 24-h (12-h) forecasts, the RMSE of radial velocity of the ASDA experiment is smaller than that of the 4DVAR experiment in Cases 2, 3, 5, 9, and $10(2,5$, $6,8,9$, and 10) although the difference is not large. The overall forecast performance of the ASDA experiment is shown to be competitive with the 4DVAR experiment in terms of the RMSE of radial velocity.

The QPF skill of the ASDA experiment is improved compared to that of the 4DVAR experiment in Cases 3, 5, and 6, and it is comparable to the 4DVAR experiment in Cases 4 and 9. The threat score of the ASDA experiment is lower than that of the CONTROL experiment in Cases 2, 7, 8, and 10 although it is higher than that of the 4DVAR experiment in Cases 7 and 8. In order to investigate the dependence of the QPF skill of the ASDA 

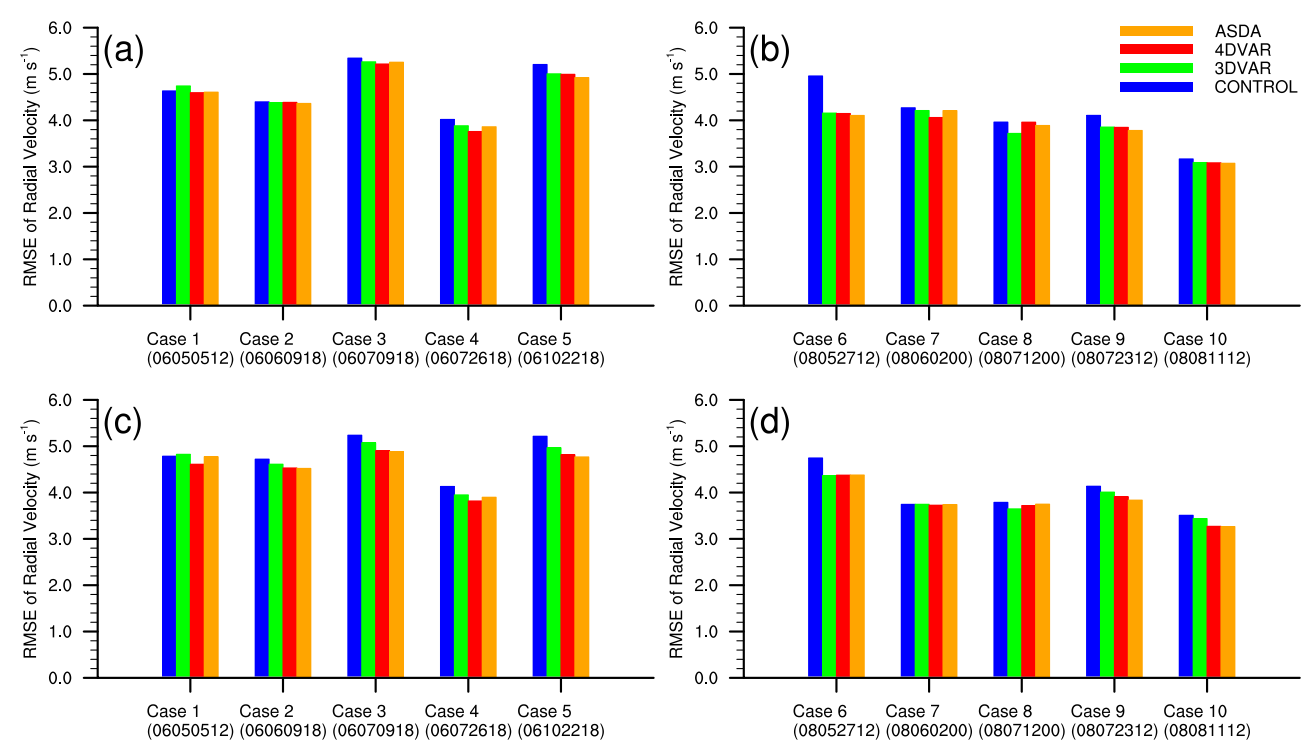

Fig. 3. RMSEs of radial velocity ( $\mathrm{m} \mathrm{s}^{-1}$ ) for the CONTROL (blue), 3DVAR (green), 4DVAR (red), and ASDA (orange) experiments. RMSEs of radial velocity were calculated using radar radial velocity observations at heights between $3 \mathrm{~km}$ and $7 \mathrm{~km}$ at an interval of 1 hour, and RMSEs during 12 -h/24-h forecasts were averaged. The averaged RMSEs over the 12-h forecasts for (a) Cases 1-5 and (b) Cases 6-10, and those over the 24-h forecasts for (c) Cases $1-5$ and (d) Cases 6-10.
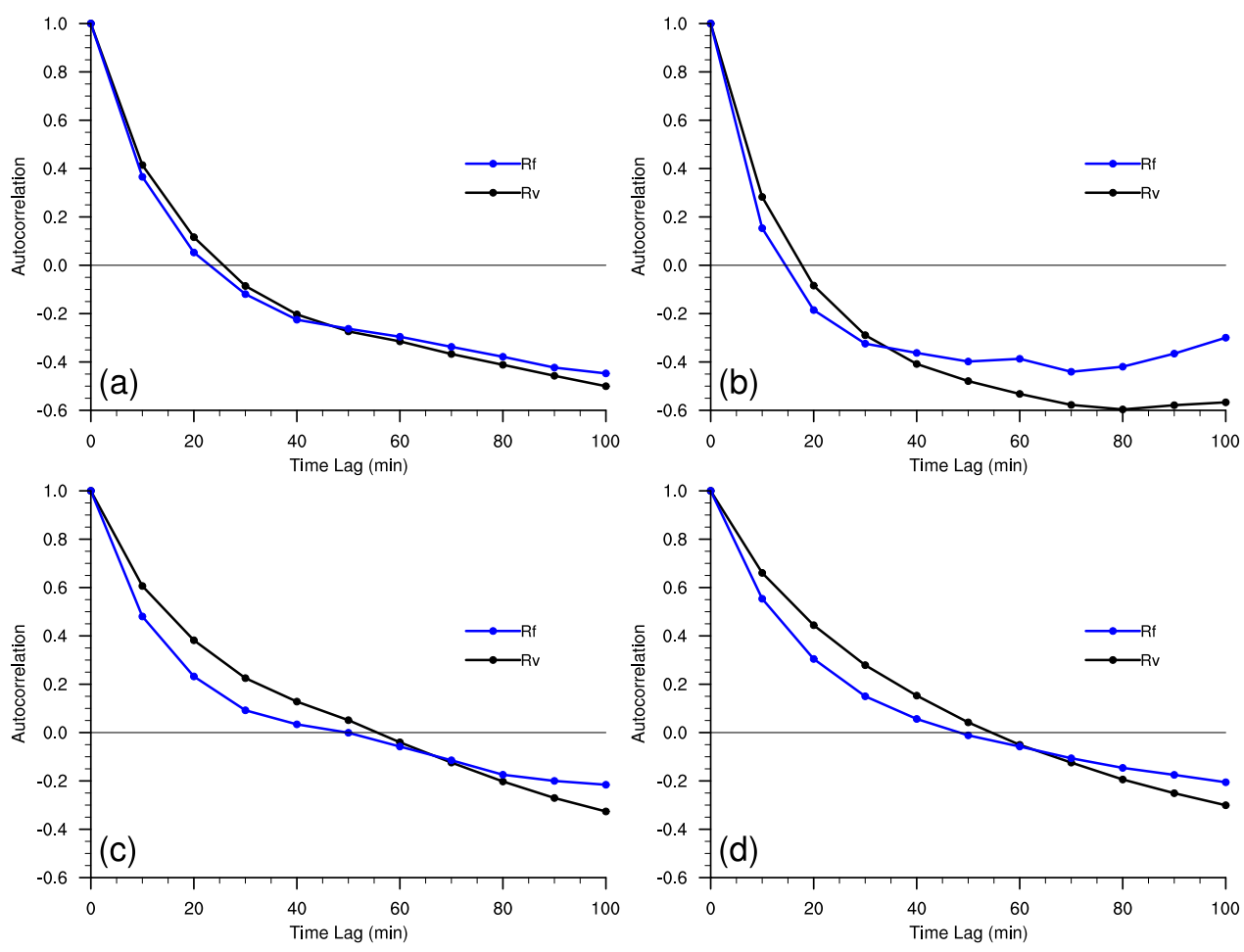

Fig. 4. Time-lagged autocorrelation coefficients of radar radial velocity (black) and reflectivity (blue) data for (a) IS, (b) SL, (c) CB, and (d) CC-type HPS.

experiment on the characteristics of heavy rainfall cases, timelagged autocorrelation coefficients of the observed radar radial velocity and reflectivity were calculated (Fig. 4). In detail, data points, where radial velocity and reflectivity data were available consistently for six hours, were selected, and a time-lagged autocorrelation of each point was calculated. Finally, the autocorrelations from the data points were averaged. The time-lagged autocorrelation can give information on the average lifetime of convective cells for each heavy rainfall case. The heavy rainfall cases selected in this study were categorized as IS, CB, CC, or SL-type Heavy Precipitation System (HPS) according to their phe- nomenological properties (Table 2). Cases 1 and 6 are classified as CC-type HPS, and Cases 2, 7, and 8 are classified as SL-type HPS. Cases 4 and 9 are categorized as CB-type HPS, and Case 10 is categorized as IS-type HPS. Heavy rainfall in Case 5 is caused by both CC- and IS-type HPSs, and Case 3 is related to typhoon EWINIAR (2006).

The time-lagged autocorrelation of radial velocity (reflectivity) for the IS-type HPS (i.e., Case 10) drops to zero after approximately 25 (20) minutes (Fig. 4a). The time-lagged autocorrelations of radial velocity and reflectivity for the SL-type HPS (i.e., the average of Cases 2, 7, and 8) decrease rapidly and reach zero after 
Table 2. Summary of heavy rainfall cases over the Korean Peninsula occurred in 2006 and 2008.

\begin{tabular}{|c|c|c|c|c|}
\hline & Analysis time & $\begin{array}{l}\text { Maximum point of } \\
\text { 24-h accumulated rainfall }\end{array}$ & $\begin{array}{l}\text { 24-h accumulated rainfall } \\
\text { amount at the maximum }\end{array}$ & HPS type \\
\hline Case 1 & 12 UTC 05 May 2006 & Ganghwa & $153.0 \mathrm{~mm}$ & $\mathrm{CC}$ \\
\hline Case 2 & 18 UTC 09 June 2006 & Seosan & $95.5 \mathrm{~mm}$ & SL \\
\hline Case 3 & 18 UTC 09 July 2006 & Namhae & $264.5 \mathrm{~mm}$ & Typhoon \\
\hline Case 4 & 18 UTC 26 July 2006 & Hongcheon & $189.0 \mathrm{~mm}$ & $\mathrm{CB}$ \\
\hline Case 5 & 18 UTC 22 October 2006 & Gangneung & $292.5 \mathrm{~mm}$ & IS \& CC \\
\hline Case 6 & 12 UTC 27 May 2008 & Seogwipo & $214.5 \mathrm{~mm}$ & $\mathrm{CC}$ \\
\hline Case 7 & 00 UTC 02 June 2008 & Seoul & $80.5 \mathrm{~mm}$ & SL \\
\hline Case 8 & 00 UTC 12 July 2008 & Hongcheon & $108.5 \mathrm{~mm}$ & SL \\
\hline Case 9 & 12 UTC 23 July 2008 & Paju & $282.5 \mathrm{~mm}$ & $\mathrm{CB}$ \\
\hline Case 10 & 12 UTC 11 August 2008 & Yanggu & $121.5 \mathrm{~mm}$ & IS \\
\hline
\end{tabular}

about 20 and 15 minutes respectively (Fig. 4b). The time required for dropping of the autocorrelation of radial velocity (reflectivity) to zero is approximately 55 (50) minutes for the CB-type HPS (i.e., the average of Cases 4 and 9; Fig. 4c). Similarly, the timelagged autocorrelation of radial velocity (reflectivity) for the CC-type HPS (i.e., the average of Cases 1 and 6) declines to zero after about 55 (50) minutes (Fig. 4d). Cases 3 and 5 are excluded from the calculation of the time-lagged autocorrelation because Case 3 is related to a typhoon, and Case 5 cannot be classified as a single-type HPS. In the cases classified as the IS- or SL-type HPS, where the average lifetime of convective storm cells is less than 30 minutes, the QPF skill of the ASDA experiment is not better than that of the CONTROL experiment. In contrast, in the cases classified as the CB- or CC-type HPS, where the duration of convective cells is greater than 30 minutes, the QPF skill of the ASDA experiment is comparable to, or better than that of the 4DVAR experiment. In the ASDA method, the observation at the end of the assimilation window is used in the computation of the adjoint sensitivity of forecast error, and the observation at the beginning of the assimilation window is used in the final 3D-Var analysis. However, the observations in between are partially used to determine the optimal value of the scaling factor. This characteristic of the ASDA method may lead to comparatively poor forecasts in cases where the average lifetime of convective cells in a HPS is relatively short (in this study, less than 30 minutes) and the nonlinearity is relatively large (discussions on the linearity assumption will be given near the end of this section). The assimilation window for the ASDA method should be determined in consideration of the characteristics of cases, availability of the observations, and computing resources. In Case 3, the QPF skill of the ASDA experiment is better than that of the 4DVAR experiment because the synoptic to meso- $\alpha$ scale forcing associated with the typhoon is dominant.

The domain mean of absolute surface pressure tendency was calculated at an interval of five minutes for each case, and they were averaged (Fig. 5). This value can be used to measure the removal of spurious high-frequency noises from the initial condition, and it is defined as follows (Lynch and Huang 1992; Lee and Lee 2003).

$$
\frac{1}{M N} \sum_{i=1}^{M} \sum_{j=1}^{N}\left|\frac{\partial p_{s}}{\partial t}\right|_{i j}
$$

where $M$ and $N$ are the number of grid points in the zonal and meridional directions, respectively. The imbalance quantified by the domain mean of absolute surface pressure tendency is larger in the 3DVAR experiment than in the 4DVAR or ASDA experiment during the first $6-\mathrm{h}$ model integration. In the 3DVAR experiment, radar radial velocity data are assimilated every 10 minutes during 30 minutes (i.e., four cycles) without any initialization, and hence the imbalance retained in the initial condition is relatively large. On the other hand, the gravity wave activity is suppressed in the 4DVAR experiment. Although the imbalance near the initial time in the ASDA experiment is greater than that in the 4DVAR experiment, the imbalance is reduced consistently, and the imbalance

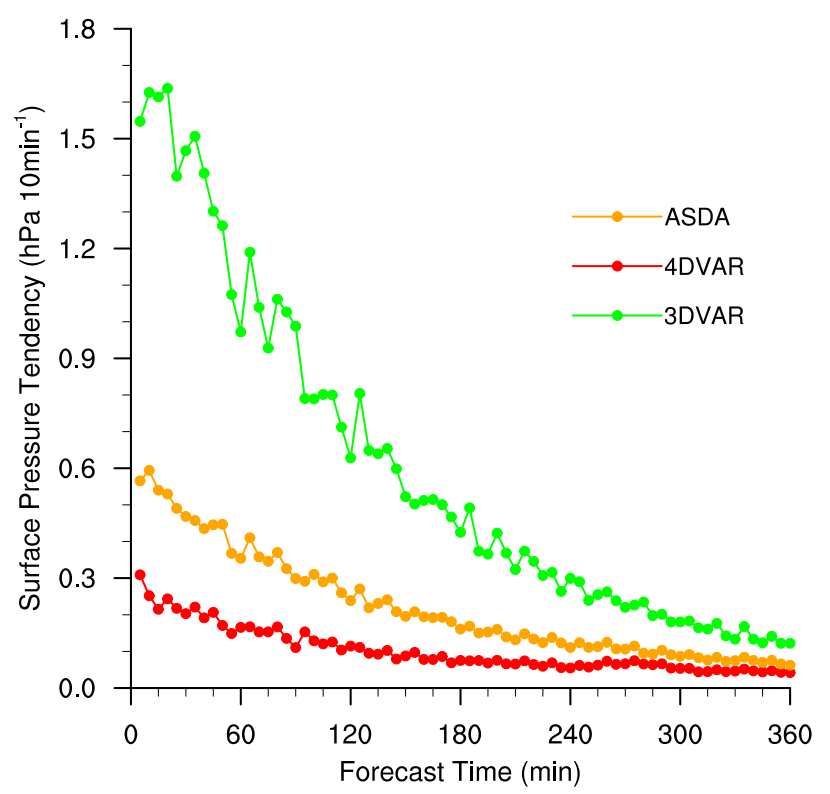

Fig. 5. Time variation of domain-averaged absolute value of surface pressure tendency (hPa $10 \mathrm{~min}^{-1}$ ) for the 3DVAR (green), 4DVAR (red), and ASDA (orange) experiments. Domain-averaged absolute surface pressure tendencies were computed for all cases, and they were averaged.

after the 4-h forecast in the ASDA experiment is similar to that in the 4DVAR experiment. In the 4DVAR and ASDA experiments, a forecast model is used as a constraint, and this leads to the analysis (or initial condition) being well-balanced with the forecast model.

In order to verify the linearity assumption on which the 4D-Var and ASDA methods are based, the ratio of linearly and nonlinearly evolved perturbation amplitudes and correlation were calculated following Kim and Jung (2009). The perturbations were calculated from analysis increments of the 4D-Var and ASDA methods, and they were evolved for 30 minutes. The ratios and correlations from 10 cases were averaged. The ratios (correlations) for zonal wind, meridional wind, and temperature are about 1.03 (0.92), $1.04(0.92)$, and $1.07(0.88)$, respectively. It is noted that the linear approximation remains valid during the assimilation window (i.e., 30 minutes) in terms of both the amplitude ratio and correlation. We also calculated the averaged ratio and correlation only for Cases 2, 7, 8, and 10 where the average lifetime of convective cells is relatively short. The ratios (correlations) for zonal wind, meridional wind, and temperature are approximately 0.89 (0.92), 0.91 (0.91), and 0.84 (0.83), respectively. Although the linear approximation still remains valid for these cases, it is not as good as for all 10 cases. The role of in-between observations seems to be important for these cases.

As a final remark, actual computing time on a LINUX cluster, which has 8 cores and 8 -GB memory, is presented. Computing 
time for a single iteration of the minimization of the 4D-Var cost function is approximately 30 minutes. Generally, about 30-60 iterations (depending on the case) are required for the convergence of the 4D-Var cost function, and hence about 15-30 hours are required for the 4D-Var method. Computational time for the 3D-Var method is much smaller than that for the 4D-Var method. Several tens of iterations for the minimization of the 3D-Var cost function take less than 5 minutes on the same machine, and hence computing time for four cycles is less than 30 minutes. This is because a single iteration for the minimization of the 4D-Var cost function involves runs of nonlinear, tangent linear, and adjoint models. Finally, the total computational cost for the ASDA method is less than one hour because the ASDA method does not require iterative minimization of the cost function.

\section{Summary and conclusions}

In this study, the ASDA method proposed in Choi et al. (2014) was applied to 10 heavy rainfall cases over the Korean Peninsula to obtain statistical robustness of the conclusions of Choi et al. (2014). We selected 10 heavy rainfall cases occurred in 2006 or 2008, and each case was classified as IS, CB, CC, or SL-type HPS according to Lee and Kim (2007). We assimilated radar radial velocity data using the (cycling) 3D-Var, 4D-Var, and ASDA methods, and made a $24-\mathrm{h}$ forecast using the analysis of each method.

The time-lagged autocorrelations of radar radial velocity and reflectivity for four types of HPSs were calculated to estimate the average lifetime of convective cells in each HPS. In cases where the average lifetime of convective cells is less than 30 minutes (i.e., cases categorized as the IS or SL-type HPS), the QPF skill of the ASDA method is not better than that of the 3D-Var or 4D-Var method. On the contrary, in cases where the average lifespan of convective cells is greater than 30 minutes (i.e., cases categorized as the CB or CC-type HPS), the QPF skill of the ASDA method is better than, or comparable to that of the 4D-Var method. In future work, we will combine the ASDA method with the Quasi Static Variational Assimilation (QSVA) method (Pires et al. 1996) to fully use the in-between observations.

In conclusion, the ASDA method is shown to be useful in assimilating radar data for various types of heavy rainfall over the Korean Peninsula. In the ASDA method, flow-dependent background error covariance is used as shown in the single observation experiment of Choi et al. (2014), and the analysis is well-balanced with a forecast model as shown in this study. It is also noted that the computational cost is reduced in the ASDA method compared to the 4D-Var method.

\section{Acknowledgements}

This work was funded by the Korea Meteorological Administration Research and Development Program under Grant CATER 2012-6080. This research was also supported by the Brain Korea 21 Plus Project in 2014.

\section{References}

Barker, D. M., and co-authors, 2012: The Weather Research and Forecasting (WRF) model's community variational/ensemble data assimilation system: WRFDA. Bull. Amer. Meteor. Soc., 93, 831-843.

Choi, Y., G.-H. Lim, D.-K. Lee, and X.-Y. Huang, 2014: An adjoint sensitivity-based data assimilation method and its comparison with existing variational methods. Tellus, 66, 21584.

Dudhia, J., 1989: Numerical study of convection observed during the winter monsoon experiment using a mesoscale twodimensional model. J. Atmos. Sci., 46, 3077-3107.

Gelaro, R., R. Buizza, T. N. Palmer, and E. Klinker, 1998: Sen- sitivity analysis of forecast errors and the construction of optimal perturbations using singular vectors. J. Atmos. Sci., 55, 1012-1037.

Hong, S.-Y., and J.-O. Lim, 2006: The WRF single-moment 6-class microphysics scheme (WSM6). J. Korean Meteor. Soc., 42, 129-151.

Hong, S.-Y., Y. Noh, and J. Dudhia, 2006: A new vertical diffusion package with an explicit treatment of entrainment processes. Mon. Wea. Rev., 134, 2318-2341.

Huang, X.-Y., N. Gustafsson, and E. R. Källén, 1997: Using an adjoint model to improve an optimum interpolation-based data-assimilation system. Tellus, 49A, 161-176.

Huang, X.-Y., and co-authors, 2009: Four-dimensional variational data assimilation for WRF: Formulation and preliminary results. Mon. Wea. Rev., 137, 299-314.

Jung, B.-J., and H. M. Kim, 2009: Moist adjoint-based forecast sensitivities for a heavy snowfall event over the Korean Peninsula on 4-5 March 2004. J. Geophys. Res., 114, D15104.

Kain, J. S., 2004: The Kain-Fritsch convective parameterization: An update. J. Appl. Meteor., 43, 170-181.

Kim, H. M., and B.-J. Jung, 2009: Influence of moist physics and norms on singular vectors for a tropical cyclone. Mon. Wea. Rev., 137, 525-543.

Kim, H. M., M. C. Morgan, and R. E. Morss, 2004: Evolution of analysis error and adjoint-based sensitivities: Implications for adaptive observations. J. Atmos. Sci., 61, 795-812.

Lee, M.-S., and D.-K. Lee, 2003: An application of a weakly constrained 4DVAR to satellite data assimilation and heavy rainfall simulation. Mon. Wea. Rev., 131, 2151-2176.

Lee, T.-Y., and Y.-H. Kim, 2007: Heavy precipitation systems over the Korean Peninsula and their classification. J. Korean Meteor. Soc., 43, 367-396.

Lynch, P., and X.-Y. Huang, 1992: Initialization of the HIRLAM model using a digital filter. Mon. Wea. Rev., 120, 1019-1034.

Mlawer, E. J., S. J. Taubman, P. D. Brown, M. J. Iacono, and S. A. Clough, 1997: Radiative transfer for inhomogeneous atmospheres: RRTM, a validated correlated-k model for the longwave. J. Geophys. Res., 102, 16663-16682.

Park, S.-G., and D.-K. Lee, 2009: Retrieval of high-resolution wind fields over the Southern Korean Peninsula using the Doppler weather radar network. Wea. Forecasting, 24, 87103.

Parrish, D. F., and J. Derber, 1992: The National Meteorological Center's spectral statistical-interpolation analysis system. Mon. Wea. Rev., 120, 1747-1763.

Pires, C., R. Vautard, and O. Talagrand, 1996: On extending the limits of variational assimilation in nonlinear chaotic systems. Tellus, 48A, 96-121.

Skamarock, W. C., J. B. Klemp, J. Dudhia, D. O. Gill, D. M. Barker, M. Duda, X.-Y. Huang, W. Wang, and J. G. Powers, 2008: A description of the Advanced Research WRF version 3. NCAR Tech. Note TN-475+STR, $113 \mathrm{pp}$.

Talagrand, O., 1997: Assimilation of observations, an introduction. J. Meteor. Soc. Japan, 75, 191-209.

Tewari, M., F. Chen, W. Wang, J. Dudhia, M. A. LeMone, K. Mitchell, M. Ek, G. Gayno, J. Wegiel, and R. H. Cuenca, Implementation and verification of the unified $\mathrm{NOAH}$ land surface model in the WRF model. 20th Conference on Weather Analysis and Forecasting/16th Conference on Numerical Weather Prediction, pp. 11-15.

Thépaut, J.-N., P. Courtier, G. Belaud, and G. Lemaître, 1996: Dynamical structure functions in a four-dimensional variational assimilation: A case study. Quart. J. Roy. Meteor. Soc., 122, 535-561.

Xiao, Q., Y.-H. Kuo, J. Sun, W.-C. Lee, E. Lim, Y.-R. Guo, and D. M. Barker, 2005: Assimilation of Doppler radar observations with a regional 3DVAR system: Impact of Doppler velocities on forecasts of a heavy rainfall case. J. Appl. Meteor., 44, 768-788.

Manuscript received 31 December 2014, accepted 24 March 2015 SOLA: https://www.jstage.jst.go.jp/browse/solal 